\title{
Matrix Transformations on Some Difference Sequence Spaces
}

\author{
Z. U. Siddiqui, A. Kiltho \\ Department of Mathematics and Statistics, University of Maiduguri, Nigeria
}

\begin{abstract}
The sequence spaces $l_{\infty}(u, v, \Delta), c_{0}(u, v, \Delta)$ and $c(u, v, \Delta)$ were recently introduced. The matrix classes $(c(u, v, \Delta): c)$ and $\left(c(u, v, \Delta): l_{\infty}\right)$ were characterized. The object of this paper is to further determine the necessary and sufficient conditions on an infinite matrix to characterize the matrix classes $(c(u, v, \Delta): b s)$ and $\left(c(u, v, \Delta): l_{p}\right)$. It is observed that the later characterizations are additions to the existing ones.
\end{abstract}

Keywords- Difference operators, Duals, Generalized weighted mean, Matrix transformations

\section{Introduction}

The sequence spaces $l_{\infty}(\Delta), c_{0}(\Delta)$ and $c(\Delta)$ were first introduced by Kizmaz [6] in 1981. Similar to the sequence spaces $l_{\infty}(p), c_{0}(p)$ and $c(p)$ for $p_{k}>1$ of Maddox [7] and Simons [10], the $\Delta$ - sequence spaces above were extended to $\Delta l_{\infty}(p), \Delta c_{0}(p)$ and $\Delta c(p)$ by Ahmad and Mursaleen [1] in ... The concept of difference operators has been discussed and used by Polat and Başar [8] and by Altay and Başar [2], both in 2007.

The idea of generalized weighted mean was applied by Altay and Başar [3], in 2006. This concept depends on the idea of $G(u, v)$ - transforms which has been used by Polat, et al [10] and by Basarir and Kara [4]. We shall need the following sequence spaces:

$\omega=\left\{x=\left(x_{k}\right): x\right.$ is any sequence $\}$

$c=\left\{x=\left(x_{k}\right) \in \omega: x_{k}\right.$ converges, i.e. $\lim _{k \rightarrow \infty} x_{k}$ exists $\}$

$c_{0}=\left\{x=\left(x_{k}\right) \in \omega: \lim _{\mathrm{k} \rightarrow \infty} x_{k}=0\right\}$, the set of all null sequences

$l_{\infty}=m=\left\{x=\left(x_{k}\right) \in \omega:\|x\|_{\infty}=\sup _{n}\left|x_{k}\right|<\infty\right\}$

$l_{1}=l=\left\{x=\left(x_{k}\right) \in \omega:\|x\|_{1}=\sum_{k=0}^{\infty}\left|x_{k}\right|<\infty\right\}$

$l_{p}=\left\{x=\left(x_{k}\right) \in \omega:\|x\|_{p}=\sum\left|x_{k}\right|^{p}<\infty ; 1 \leq p<\infty\right\}$

$\phi=\left\{x=\left(x_{k}\right) \in \omega: \exists N \in \mathbb{N} / 8\right.$ uch that $\left.\forall k \geq N, x_{k}=0\right\}$, the set of finitely non-

$b s=\left\{x=\left(x_{k}\right) \in \omega:\|x\|_{b s}=\sup _{n}\left|\sum_{k=0}^{n} x_{k}\right|<\infty\right\}$, the set of all sequences with bounded partial sums

$X^{\beta}=\left\{a=\left(a_{k}\right) \in \omega: \sum_{k=0}^{\infty} a_{k} x_{k} \in c, \forall x \in X\right\}$

Note that $x=\left(x_{k}\right)$ is used throughout for the convention $\left(x_{k}\right)=\left(x_{k}\right)_{k=0}^{\infty}$. We take $e=(1,1,1, \ldots)$ and $e^{k}$ for the sequence whose only nonzero term is 1 in the $k$ th place for each $k \in \mathbb{N}$, where $\mathbb{N}=\{0,1,2,3, \ldots\}$. Any vector subspace of $\omega$ is called a sequence subspace. A sequence space $X$ is $F K$ if it is a complete linear metric space with continuous coordinates $P_{n}: X \rightarrow \mathbb{C}$, defined by $P_{n}(x)=x_{n} \forall x=\left(x_{k}\right) \in X$ with $n \in \mathbb{N}$. A normed $F K$ space is $B K$-space or Banach space with continuous coordinates. An $F K$ space has $A K$ - property if $x^{[m]} \rightarrow x$ in $X$, where $x^{[m]}=\sum_{k=0}^{n} x_{k} e^{k}$ is the $\mathrm{m}^{\text {th }}$. section of $x$. If $\varphi$ is dense in $X$ then it has an $A D$ - property (see Boos [5]). A matrix domain of a sequence space $X$, is defined as $X_{A}=\left\{x=\left(x_{k}\right) \in \omega: A x \in X\right\}$.

Let $\mathcal{U}$ be the set of all sequences $u=\left(u_{k}\right)$ with $u_{k} \neq 0 \forall k \in \mathbb{N}$, and for $u \in U$ let $\frac{1}{u}=\left(\frac{1}{u_{k}}\right)$. Then for $u, v \in \mathcal{U}$ define the matrix $G(u, v)=\left(g_{n k}\right)$ by

$$
g_{n k}=\left\{\begin{array}{l}
u_{n} v_{k}, \text { for } 0 \leq k \leq n, \\
0, \quad \text { for } k>n \quad \forall k, n \in \mathbb{N}
\end{array}\right.
$$

This matrix is called the generalized weighted mean. The sequence $y=\left(y_{k}\right)$ in the sequence spaces

$$
\lambda(u, v, \Delta)=\left\{x=\left(x_{k}\right) \in \omega: y=\sum_{i=0}^{k} u_{k} v_{i} \Delta x_{i} \in X\right\}, \lambda \in\left\{l_{\infty}, c, c_{0}\right\}
$$

is the $G(u, v, \Delta)-$ transform of a given sequence $x=\left(x_{k}\right)$. It is defined by

where,

$$
\begin{aligned}
y & =\sum_{i=0}^{k} u_{k} v_{i} \Delta x_{i} \\
& =\sum_{i=0}^{k} u_{k} \nabla v_{i} x_{i}
\end{aligned}
$$

$$
\nabla v_{i}=v_{i}-v_{i+1} \text { and } \Delta x=\left(\Delta x_{i}\right)=x_{i}-x_{i-1},
$$

and taking all negative subscripts to be naught. The spaces (1) were defined in [9]. If $X$ is any normed sequence space the matrix domain $X_{G(u, v, \Delta)}$ is the generalized weighted mean difference sequence space [9]. Our object is to characterize the matrix classes $\left(c(u, v, \Delta): l_{p}\right)$ and $(c(u, v, \Delta): b s)$. However, matrix class characterizations are done with help of $\beta$-duals, and so we need the following 
Lemma 1.1 [9]: Let $u, v, \in \mathcal{U}, a=\left(a_{k}\right) \in \omega$ and the matrix $D=\left(d_{n k}\right)$ by

$$
d_{n k}=\left\{\begin{array}{l}
\left(\frac{1}{u_{n} v_{k}}-\frac{1}{u_{n} v_{k+1}}\right) a_{k} ; \quad(0 \leq k<n), \\
\frac{1}{u_{n} v_{n}} a_{n} ; \quad(k=n) \\
0 ; \quad(k>n)
\end{array}\right.
$$

and let $d_{1}, d_{2}, d_{3}, d_{4}$ and $d_{5}$ be the sets

$$
\begin{aligned}
& d_{1}=\left\{a=\left(a_{k}\right) \in \omega: \sup _{n} \sum_{n}\left|\sum_{k \in \mathcal{K}} d_{n k}\right|<\infty\right\} ; \\
& d_{2}=\left\{a=\left(a_{k}\right) \in \omega: \sup _{n} \sum_{n}\left|d_{n k}\right|<\infty\right\} ; \\
& d_{3}=\left\{a=\left(a_{k}\right) \in \omega: \lim _{n \rightarrow \infty} d_{n k} \text { exists for each } n \in \mathbb{N}\right\}
\end{aligned}
$$

Then, $\left[c_{0}(u, v, \Delta)\right]^{\beta}=d_{1} \cap d_{2} \cap d_{3}$.

\section{Methodology}

If $A$ is an infinite matrix with complex entries $a_{n k}(n, k \in \mathbb{N})$, then $A=\left(a_{n k}\right)$ is used for $A=$ $\left(a_{n k}\right)_{n, k=0}^{\infty}$ and $A_{n}$ is the sequence in the $\mathrm{n}^{\text {th }}$ row of $A$, or $A_{n}=\left(a_{n k}\right)_{k=0}^{\infty}$ for every $n \in \mathbb{N}$. The $A$ - transform of a sequence $x$ is defined as

$$
\begin{aligned}
A x & =\left(A_{n}(x)\right)_{n=0}^{\infty} \\
& =\lim _{n \rightarrow \infty} \sum_{k=0}^{\infty} a_{n k} x_{k} \quad(n \in \mathbb{N})
\end{aligned}
$$

provided the series on the right converges for each $n$ and for all $x \in X$. The pair $(X, Y)$ is referred to as a matrix class, so that

$$
A \in(X, Y) \Leftrightarrow\left\{\begin{array}{l}
A_{n} \in X^{\beta} \forall n \in \mathbb{N} \\
\text { and } \\
A x \in Y \quad \forall x \in X, \text { in the norm of } Y
\end{array}\right.
$$

In this paper we shall take $X=c(u, v, \Delta)$ and $Y \in\left\{l_{p}, b s\right\}$. We shall need the following lemma for the proof of Theorems 3.1 and 3.2 as our main results in section 3:

Lemma 2.1 [9]: The sequence spaces $\lambda(u, v, \Delta)$ for $\lambda \in\left\{l_{\infty}, c, c_{0}\right\}$ are complete normed linear spaces with the norm $\|x\|_{\lambda(u, v, \Delta)}=\sup _{k}\left|\sum_{i=0}^{k} u_{k} \Delta x_{i}\right|=\|y\|_{\lambda}$. They are also $B K$ spaces with both $A K$ - and $A D$ - properties. Further, let $y \in c_{0}$ and define $x=\left(x_{k}\right)$ by

then $x \in c_{0}(u, v, \Delta)$.

$$
x_{k}=\sum_{i=0}^{k-1} \frac{1}{u_{k}}\left(\frac{1}{v_{i}}-\frac{1}{v_{i+1}}\right) y_{i}+\frac{1}{u_{k} v_{k}} y_{k} ; k \in \mathbb{N}
$$

An infinite matrix $A$ maps a $B K$ space $X$ continuously into the space $b s$ if and only if the sequence the sequence of functional $\left\{f_{n}\right\}$ defined by

$$
f_{n}(x)=\sum_{n=1}^{m} \sum_{k=1}^{\infty} a_{n k} x_{k}, \quad n=1,2,3, \ldots
$$

is bounded in the dual space of $X$.

\section{Main Results}

Theorem 3.1. $A \in\left(c(u, v, \Delta): l_{p}\right)$ for $p>1$, if and only if

$$
\begin{aligned}
& \sup _{n}\left|\sum_{k \in \mathcal{K}}\left[\sum_{i=1}^{k-1} \frac{1}{u_{k}}\left(\frac{1}{v_{i}}-\frac{1}{v_{i+1}}\right) a_{n k}+\frac{1}{u_{k} v_{k}} a_{n k}\right]\right|^{p}<\infty, \\
& \lim _{n \rightarrow \infty}\left[\sum_{i=1}^{k-1} \frac{1}{u_{k}}\left(\frac{1}{v_{i}}-\frac{1}{v_{i+1}}\right) a_{n k}+\frac{1}{u_{k} v_{k}} a_{n k}\right]=a_{k}, \text { exists } \\
& \lim _{n \rightarrow \infty} \sum_{k=0}^{n}\left[\sum_{i=1}^{k-1} \frac{1}{u_{k}}\left(\frac{1}{v_{i}}-\frac{1}{v_{i+1}}\right) a_{n k}+\frac{1}{u_{k} v_{k}} a_{n k}\right]=a, \text { exists }
\end{aligned}
$$

Proof: Since $c(u, v, \Delta)$ and $l_{p}$ are $B K$ spaces, we suppose that (i), (ii) and (iii) hold and take $x=\left(x_{k}\right) \in$ $c(u, v, \Delta)$. Then by (2) and Lemma 1.1, $A_{n} \in[c(u, v, \Delta)]^{\beta}$ for all $n \in \mathbb{N}$, which implies the existence of the $A$ transform of $x$, or $A x$ exists for each $n$. It is also clear that the associated sequence $y=\left(y_{k}\right)$ is in $c$ and hence $y \in c_{0}$. Again, since $c(u, v, \Delta)$ has $A K$ (Lemma 2.1) and contains $\phi$, by the $\mathrm{m}^{\text {th }}$ partial sum of the series $\sum_{k=0}^{\infty} a_{n k} x_{k}$ we have

$$
\sum_{k=0}^{m} a_{n k} x_{k}=\sum_{k=0}^{m}\left[\sum_{i=1}^{k-1} \frac{1}{u_{k}}\left(\frac{1}{v_{i}}-\frac{1}{v_{i+1}}\right)+\frac{1}{u_{k} v_{k}}\right] a_{n k} y_{k}
$$

which becomes

$$
\sum_{k=0}^{\infty} a_{n k} x_{k}=\sum_{k=0}^{\infty}\left[\sum_{i=1}^{k-1} \frac{1}{u_{k}}\left(\frac{1}{v_{i}}-\frac{1}{v_{i+1}}\right) a_{n k}+\frac{1}{u_{k} v_{k}} a_{n k}\right] y_{k}, \text { for } p>1
$$




$$
\begin{aligned}
\Rightarrow\|A x\|_{l_{p}} & \leq \sup _{n} \sum_{k}\left[\sum_{k=0}^{k-1}\left|\frac{1}{u_{k}}\left(\frac{1}{v_{i}}-\frac{1}{v_{i+1}}\right) a_{n k} y_{k}+\frac{1}{u_{k} v_{k}} a_{n k} y_{k}\right|^{p}\right]^{1 / p} \\
& \leq\left\|y_{k}\right\|_{l_{p}} \sup _{n}\left(\sum_{k}\left[\sum_{k=0}^{k-1}\left|\frac{1}{u_{k}}\left(\frac{1}{v_{i}}-\frac{1}{v_{i+1}}\right) a_{n k}\right|^{p}\right]^{1 / p}+\left[\sum_{k=0}^{k-1}\left|\frac{a_{n k}}{u_{k} v_{k}}\right|^{p}\right]^{1 / p}\right)<\infty
\end{aligned}
$$

$\Rightarrow A x \in l_{p}$ and hence $A \in\left(c(u, v, \Delta): l_{p}\right)$.

Conversely, let $A \in\left(c(u, v, \Delta): l_{p}\right), 1<p<\infty$. Then again by (2) and Lemma 1.1, $A_{n} \in[c(u, v, \Delta)]^{\beta}$ for all $n \in \mathbb{N}$ implying (ii) and (iii) for all $x \in c(u, v, \Delta)$ and $y \in l_{p}$. To prove (i), let the continuous linear functional $f_{n}(n \in \mathbb{N})$ be defined on $(c(u, v, \Delta))^{*}$, the continuous dual of $c(u, v, \Delta)$. Since the series $\sum_{k=0}^{\infty} a_{n k} x_{k}$ converges for each $x$ and for each $n$, then $f_{A_{n}} \in(c(u, v, \Delta))^{*}$; where

$$
\begin{aligned}
& f_{A_{n}}(x)=\sum_{k=0}^{\infty} a_{n k} x_{k} \forall x \in c(u, v, \Delta) . \\
& \Rightarrow\left\|f_{A_{n}}\right\|=\left\|A_{n}\right\|_{l_{p}}=\left(\sum_{k=0}^{\infty}\left|a_{n k}\right|^{p}\right)^{\frac{1}{p}}<\infty, \text { for all } n \in \mathbb{N},
\end{aligned}
$$

with $A_{n} \in[c(u, v, \Delta)]^{\beta}$. This means that the functional defined by the rows of $A$ on $c(u, v, \Delta)$ are pointwise bounded, and by the Banach-Steinhaus theorem these functional are uniformly bounded. Hence there exists a constant $M>0$, such that $\left\|f_{A_{n}}\right\| \leq M, \forall n \in \mathbb{N}$, yielding (i).

Theorem 3.2: $A \in(c(u, v, \Delta): b s)$ if and only if conditions (ii) and (iii) of Theorem 3.1 hold, and

$$
\sup _{m} \sum_{k} \sum_{n=1}^{m}\left|\sum_{i=1}^{k-1} \frac{1}{u_{k}}\left(\frac{1}{v_{i}}-\frac{1}{v_{i+1}}\right) a_{n k}+\frac{1}{u_{k} v_{k}} a_{n k}\right|<\infty .
$$

Proof. Suppose $A \in(c(u, v, \Delta): b s)$. Then $A_{n} \in[c(u, v, \Delta)]^{\beta}$ for all $n \in \mathbb{N}$. Since $e_{k}=\left(\delta_{n k}\right)$, where $\delta_{n k}=1$ $(n=k)$ and $=0(n \neq k)$, belongs to $c(u, v, \Delta)$, the necessity of (ii) holds. Similarly by taking $x=e=$ $(1,1,1, \ldots) \in c(u, v, \Delta)$ we get (iii). We prove the necessity of (i) as follows:

Suppose $A \in(c(u, v, \Delta): b s)$. Then it implies

$$
\sum_{n=1}^{m}\left|A_{r}(x)\right|<\infty, m=1,2,3, \ldots,
$$

where,

$$
A_{r}(x)=\sum_{k} a_{r k}\left(\sum_{i=0}^{k-1}\left(\frac{y_{k}}{u_{k}}\left(\frac{1}{v_{i}}-\frac{1}{v_{i+1}}\right)+\frac{y_{k}}{u_{k} v_{k}}\right)\right)
$$

converges for each $r$ whenever $x \in c(u, v, \Delta)$, which follows by the Banach-Steinhaus theorem that $\sup _{k}\left|a_{n k}\right|<\infty$, each $r$. Hence $A_{r}$ defines an element of $[c(u, v, \Delta)]^{*}$ for each $r$.

Now define

$$
q_{m}(x)=\sum_{n=1}^{m}\left|A_{r}(x)\right|, \quad r=1,2,3, \ldots
$$

$q_{m}$ is subadditive. Moreover, $A_{r}$ is a bounded linear functional on $c(u, v, \Delta)$ implies each $q_{m}$ is a sequence of continuous seminorms on $c(u, v, \Delta)$ such that

$$
\sup _{m} q_{m}(x)=\sum_{r=1}^{\infty}\left|A_{r}(x)\right|<\infty \text { for each } x \in c(u, v, \Delta) .
$$

Thus there exists a constant $M>0$ such that

which implies (i).

$$
\sum_{r=1}^{\infty}\left|A_{r}(x)\right| \leq M\|x\|_{c(u, v, \Delta)}
$$

Sufficiency: Suppose (i) - (iii) of the theorem hold. Then $A_{n} \in[c(u, v, \Delta)]^{\beta}$. If $x \in c(u, v, \Delta)$, it suffices to show that $A_{n}(x) \in b s$ in the norm of the sequence space $b s$.

Now,

$$
\sum_{k=0}^{n} a_{n k} x_{k}=\sum_{k=0}^{n}\left[\left[\sum_{i=1}^{k-1} \frac{1}{u_{k}}\left(\frac{1}{v_{i}}-\frac{1}{v_{i+1}}\right)+\frac{1}{u_{k} v_{k}}\right] a_{n k}\right] y_{k}
$$




$$
\begin{aligned}
& \leq \sup _{n} \sum_{k=0}^{n}\left[\sum_{i=0}^{k-1}\left(\frac{1}{u_{k}}\left(\frac{1}{v_{i}}-\frac{1}{v_{i+1}}\right) a_{n k}+\frac{a_{n k}}{u_{k} v_{k}}\right) y_{k}\right] \quad \text { by (i) } \\
& \leq\left\|y_{k}\right\| \sup _{n} \sum_{k=0}^{\infty}\left[\sum_{i=1}^{k-1} \frac{1}{u_{k}}\left(\frac{1}{v_{i}}-\frac{1}{v_{i+1}}\right)+\frac{1}{u_{k} v_{k}}\right] a_{n k}<\infty, \text { as } n \rightarrow \infty .
\end{aligned}
$$

This implies $A_{n}(x) \in b s$ or $A \in(c(u, v, \Delta): b s)$.

\section{Concluding Remarks}

The generalization obtained here still admit improvement in the sense that the conditions obtained here may further be simplified resulting in less restrictions on the involved matrices.

\section{References}

[1] Ahmad, Z. U., and Mursaleen, Köthe-Teoplitz Duals of Some New Sequence Spaces and Their Matrix Transformations, Pub. Dé L'institut Mathématique Nouvelle série tome 42 (56), 1987, p. 57-61

[2] Altay, B., and F. Başar, Some Paranormed Sequence Spaces of Non-absolute Type Derived by Weighted Mean, J. math. Anal. Appl. 319, (2006), p. 494-508

[3] Altay B., and F. Başar, The Fine Spectrum and the Matrix Domain of the Difference Operator $\Delta$ on the Sequence Space $\mathrm{l}_{\mathrm{p}}$, $0<p<$ 1), Comm. Math. Anal. Vol. 2 (2), 2007, p. 1-11

[4] Başarir, M., and E. E. Kara, On Some Difference Sequence Spaces of Weighted Means and Compact Operators, Ann. Funct. Anal. 2,2011, p. 114-129

[5] Boos, J., Classical and Modern Methods in Summability, Oxford Sci. Pub., Oxford, 2000

[6] Kizmaz, H., On Certain Sequence Spaces, Canad. Math. Bull. Vol. 24 (2), 1981, p. 169-176

[7] Maddox, I. J., Spaces of Strongly Summable Sequences, Quart. J. Math. Oxford,18 (2), 1967, p. 345-355

[8] Polat, H., and F. Başar, Some Euler Spaces of Difference Sequences of Order m*, Acta Mathematica Scienta, 2007, 27B (2), p. 254266

[9] Polat, H., Vatan K. and Necip S., Difference Sequence Spaces Derived by Generalized (5), 2011, p. 608-614

Weighted Mean, App. Math. Lett. 24

[10] Simons, S., The Sequences Spaces, $\mathrm{l}\left(\mathrm{p}_{\mathrm{v}}\right)$ and $\mathrm{m}\left(\mathrm{p}_{\mathrm{v}}\right)$, Proc. London Math. Soc. 15 (3), 1965, p. $422-436$ 